\title{
Chapter n. Evaluating BCI for musical expression: historical approaches, challenges and benefits
}

Duncan A.H. Williams (orcid.org/0000-0003-4793-8330), University of York, UK.

Keywords: Music, sound, sonification, multi-criteria decision aid

\begin{abstract}
A recurring challenge in the use of $\mathrm{BCI}$ (and more generally $\mathrm{HCI}$ ) for musical expression is in the design and conduct of appropriate evaluation strategies when considering BCI systems for music composition or performance. Assessing the value of computationally assisted creativity is challenging in most artistic domains, and the assessment of computer assisted (or entirely computer generated) music is no different. BCI provides two unique possibilities over traditional evaluation strategies: firstly, the possibility of devising evaluations which do not require conscious input from the listener (and therefore do not detract from the immersive experience of performing, creating, or listening to music), and secondly in devising neurofeedback loops to actively maneuver the creator or listener through an expressive musical experience. Music offers some unusual challenges in comparison to other artistic interfaces: for example, often it is made in ensemble, and there is evidence to suggest neurophysiological differences are evident in ensemble measurement when compared to solo performance activities, for example see (Babiloni et al. 2011). Moreover, a central purpose of music is often to incite movement (swaying, nodding head, dancing) - both in performer and audience - and as such this also offers up challenges for BCI/HCI design. This chapter considers historical approaches as well as making proposals for borrowing solutions from the world of auditory display (also referred to as sonification) and psychoacoustic evaluation techniques, to propose a hybrid paradigm for the evaluation of expression in BCI music applications.
\end{abstract}




\section{n.1 Introduction}

Music has been described as a language for emotional expression (Lin and Cheng 2012) and is comprised of both communication, and interaction. Music allows communcation from the composer or performer to an audience of listener(s), and symbiotically between performer and audience, and performer(s). Music as a form of artistic expression is ubiquitously popular, perhaps because listeners need no special musical training to enjoy or understand musical expression (Bailes and Dean 2009; Bigand and Poulin-Charronnat 2006). BCI and other biophysiological sensor techniques have gradually been adopted by the research community involved in the design of new musical instruments, music information retrieval, and computationally-assisted musical creativity (for example, algorithmic composition systems, automated accompaniment systems and the like). One term gaining traction amongst the community for this field is Brain-Computer Music Interfacing, or BCMI, (Miranda and Castet 2014), though this does not tend to include the full range of possible musical experiences, and indeed multimodal sensors are more commonplace (motion tracking, galvanic skin response, heart rate measurement) than BCI alone. Computational creativity is an emerging field, and, like BCMI, does not have established methodologies for robust evaluation. Simply put, a BCI generated composition may be designed by engineers or composers, but then be unobjectively rendered, or perhaps explored with 'Turing style' testing to establish convincing algorithms. The potential use of BCI to offer meaningful and responsive control signals for music generation has yet to be fully realized, though BCI has been used by some to adapt the design of generative music techniques that respond to brain signals. For example, to offer platforms for music making to improve the lives of people with physical disabilities, as well as in the more common design of applications for artistic 
purposes.

In these contexts, BCI offers some unique possibilities over traditional music making, particularly in the design of expressive systems with emotionally-congruous mappings between brain derived control signal, and musical feature selection or performance. In the long term, this may be useful for commercial applications, functional music selection, and to provide tools for individuals with particular communicative problems to create aids for communicating emotional state (e.g., people with Aspergers syndrome).

This chapter will provide a brief review of systems for BCI and music, before considering the challenges involved in the design of such systems and the need for specific and context dependent evaluation methodologies. Therefore for our purposes we will assume that You the reader will already have experience with the vast majority of the particular BCI methods described here. Musical applications tend to borrow from and build upon existing robust strategies, rather than improve upon or develop new hardware or software methods for measurement. For example the P300 or 'oddball' paradigm, the use of steady-state visually evoked stimuli (SSVEP), asymmetry measurement and filter based techniques from electroencephalogram (EEG) measurement, as well as hybrid systems incorporating the other biophysiological measurements above, and traditional psychometric evaluation techniques (self assessment in both qualitative and quantitative domains). Here, there are advantages in terms of unconscious response, the potential for neurofeedback, and designing a sense of agency over the performance, and music specific challenges including the tendency of music to induce a motile response (dancing, head nodding), or a common tendency to be designed for ensemble performance (such as live music concerts).

Pleasingly, a number of the challenges presented by traditional evaluation of computationally assisted creativity in music might actually be solved by the use of BCI. Typically evaluation is rare, or might be simplistic (did the audience enjoy a performance, did the music 'sound 
good', did a recording sell well, or similar questions which are highly variable and subjective). Therefore we conclude with some suggestions for future evaluation strategies which borrow from the world of auditory display (often simply called sonification). There are several examples of music created by means of sonifying EEG (or other biophysiological) data, either in real time or through more complex systems.

Some suggestions for further work are also volunteered, including development of collaborative platforms for music performance by means of BCMI. The field, though small at first glance, is steadily growing, and this chapter focuses on a discrete group of research in the context of the field - inclusive but by no means exhaustive - a great variety of existing work is taking place at the time of writing. Music remains an exciting and challenging application, particularly at this time, for the BCI community.

\section{n.2 Historical review and possibilities for BCI in music making}

BCI for music making is not common amongst music technologists, instrument designers, and the like, in comparison to the large research communities actively engaged in new musical instrument or music information retrieval problems. Nevertheless the community investigating the use of BCI for music has slowly gained traction over the past two decades. Typical systems might analyse a real-time input, subject it to a range of signal processing (perhaps filtering or more complicate statistical reductions) and use the resulting signal to choose or create from scratch a musical stimulus. The potential for such systems includes provision of aesthetic communication tools through music for users who are not musically confident or trained in performance to a level where they might engage in traditional musicmaking (Clair and Memmott 2008; Fagen 1982; Hanser 1985). Engaging with music making activities has been shown to be therapeutic in the treatment of both physical and mental impairments (Aldridge 2005; Hanser 1985). 
Early pioneers made use of EEG to create contemporary music performances in concert settings, such as Alvin Lucier's 1965 Music for Solo Performer (Lucier 1976) which used a single electrode to distribute amplified alpha waves to a number of percussion instruments, which are then essentially stimulated 'hands free' by the performer, who mediates their mental state to give some degree of control over the performance itself. Richard Teitelbaum explored the use of an amplified EEG signal as a control source for analog sound synthesis in an improvised performance in the 1967 piece Spacecraft (Teitelbaum 1976). Ideas regarding the use of adaptive biofeedback in music were explored by Eaton (1971), who combined visual and auditory stimuli.

David Rosenboom was inspired by this work and continued its explorations in his Brainwave Music (1974), an interesting example as it was designed to incorporate the use of biofeedback in the performance process (Rosenboom 1990; Teitelbaum 1976).

Biomuse (Knapp and Lusted 1990) mapped the acquisition of low-level neuroelectric and myoelectric signals via statistical feature extraction to the real-time generation of music notation (musical structures in MIDI format). Biomuse also used other physiological readings (muscle tension and eye tracking). Whilst such signals are tangential to BCI, there is a growing field of work using non-nervous physiological signals, such as heart rate, galvanic skin response, and so on, in the design of systems for creative music technology (Daly et al. 2015a; Nirjon et al. 2012; Pérez and Knapp 2008). One of the earliest examples of similar work combining signals for musical performance can be seen in Richard Teitelbaum's In Tune (1967), which used two EEG inputs alongside heartbeat and breathing sensors to give the performers control of a variety of analog synthesis functions.

BCI offers the possibility of directly translating brain activity (for example, motor or visual 
cortex activity, or more abstractly, emotional state for expression) to inform performance in music making. For example, particular frequencies of brain activity could be correlated with fixed musical parameters, so that the performer is required to mediate their own brainwave frequencies to achieve the intended musical output from the system (e.g., actively attempting to mediate brainwave amplitudes and frequencies as collected by the EEG). For the purposes of this chapter, we will consider this parameter mapping (forming control signal links between established BCI metrics and musical parameters).

These parameters might be musical control signals; temporal (start or end a playback) dynamic (adjust volume) or spectral (frequency equalization) for example. An overview of specific mapping techniques for digital instrument design is given by Goudeseune (2002). More recently, an overview of different types of musical parameter mapping from complex biomedical data and possible evaluation strategies is given in Williams (2016), but design and evaluation of such mappings for maximal musical expression remains a significant area for further work at the time of writing. Various combinations of mapping strategies exist, including one-to-one, one-to-many, and many-to-many combinations (Hunt and Kirk 2000), and indeed the linear mapping of alpha waves to particular acoustic instruments in Music for Solo Performer is significantly different to the more complex mappings employed latterly, including ensemble performances in examples by the Biomuse Trio (Knapp et al. 2009; Lyon et al. 2014) (see, e.g., their 2011 piece Music for Sleeping and Waking Minds). Whilst on-thefly mapping is theoretically possible, musical parameter mapping is predetermined at the stage of system design and generally considered a part of the compositional process. It is in the mapping stage that systems for music composition generally derive their variety. Both the format of the output and the parameter selection, and ratios between control and parameter are considered valid, with many different types of mappings explored by those working with BCI for music (Brouwer and van Erp 2010; Chew and Caspary 2011; Daly et al. 2014c). 
Further opportunity for musical expression and variety can be given at the performance stage. BCI measurement has been combined with real-time sound synthesis in musical performance contexts (Hinterberger and Baier 2005). The use of BCI informed musical stimulus selection to mediate or entrain the listeners' brain activity (i.e., neurofeedback) is also a fertile area for research activity (Daly et al. 2014a, 2016a).

Recently, machine learning techniques are being used to inform hybrid adaptive processing of control signals for music generation and performance (AlZoubi et al. 2008, 2009).

Neurofeedback is particularly suitable for the specification of combined composition and performance music systems.

It is perhaps not surprising that of the biophysiological measurement techniques which are often adapted to music making, EEG is prevalent, due to the cost and accessibility of the relevant hardware. Amongst EEG based systems, both event related potentials (ERP) and spontaneous input are common. The P300 ERP (or "oddball paradigm") has been deployed in a system to allow active control over note selection for real-time synthesis (Grierson 2008; Grierson and Kiefer 2011) - techniques which are not dissimilar to the commonplace ERP typing or spelling systems, but used for the selection of musical notes rather than text input. Similarly, stimulus-responsive input measures such as the SSVEP (Middendorf et al. 2000), have been adapted to real-time control of musical parameters such as volume, or even limited selection of pre-composed score. However such systems are markedly different to approaches sonifying or musifying brainwave data (Baier et al. 2007a,b; Hinterberger and Baier 2005), wherein EEG (or other BCI data) is directly transmitted by auditory means (Toharia et al. 2014). Indeed, many existing EEG mappings for sonification are now in use (Väljamäe et al. 2013). The link comes again in the mapping between musical parameters (ruleset or other compositional decision making processes) so that the $\mathrm{BCI}$ input is constrained in some musically meaningful manner to create a performance with compositional intent, and 
aesthetic expression for the listener. One system for musifying EEG data mapped the rate of alpha wave activity to the cadence of the rhythm structure in a series of musical segments, while mapping the variance in the EEG to musical chord selections on a bar-by-bar basis, and the note position of a melody to the amplitude of the EEG waves per analysis window (Wu et al. 2010). Rhythm is an interesting musical property with specific brain cortex associations (Baier et al. 2007c) and, as such, has also been utilized in EEG analysis of musical rhythm, for example, in the evoked gamma band $(20-60 \mathrm{~Hz})$ by rhythmic tone sequences (Snyder and Large 2005). This type of mapping has also been explored in reverse, where the rhythmic properties of the resulting material are directly controlled by BCI input (Daly et al. 2014c). Making music in ensemble has a rich history (Le Groux and Verschure 2009; Manzolli and Verschure 2005), and has begun to be explored in BCI informed music making, for example a system which provides the ability for two users to collaborate in musical performance by mapping BCI measures of affect to the control of amplitude of two separate musical features (Leslie and Mullen 2012). Mullen et al also survey systems which are designed to give agency to multiple performers, which they describe as social installations (Mullen et al. 2015). This work is closely aligned with the spirit of musical performance as communicative and interactive.

\section{n.2.1 Possibilities}

In all of the systems described above there remains a separation between the use of BCI as a cognitive control (active control) or the deliberate mapping of composition or performative generative music techniques in a passive (unconscious) manner. Recent research has suggested a number of unconscious cognitive performance benefits for the listener when music which is particularly evocative is played (Franco et al. 2014). An example of affective state mapping to unconscious musical feature selection can be seen 
in the world of musical information retrieval (Lin and Cheng 2012). The potential to create systems for functional music (selection, performance, or even creation) in an unconscious manner (i.e., without the need for active management by the user) is enormous and perhaps the largest likely avenue for BCI music creation in terms of broad user base. Levels of emotional engagement, as measured via BCI, have been adapted to musical control by Ramirez and Vamvakousis (2012). They analysed EEG recordings elicited from listeners who were played a database of music which they considered to be emotionally charged, across a two-dimensional affect space (a commonly used space in psychometric evaluation, the arousal-valence, or circumplex model, of affect), defining affective (emotional) states from EEG (Chanel et al. 2006, 2007). For the original source of the circumplex model the interested reader is referred to (Russell 1980). The overarching tendency is to spend time creating complicated mappings but not exploring how successful these were in communicating artistic or aesthetic intent - which, perhaps ironically, is one of the most promising areas of BCI in the arts (as explored elsewhere in this book) as a tool for evaluating aesthetic experience, —in other words, an emotional response to music (Lin et al. 2010).

\section{n.2.2 Overcoming the self-report confound}

In music psychology, a great deal of attention has been paid to determining listeners emotional responses to certain types of music. This has significant implications for the use of BCI in evaluation of music. For example, "sad" music - or music which listeners report to communicate sadness - has been shown to be enjoyable (Vuoskoski and Eerola 2012; Vuoskoski et al. 2012) and subsequently, to have similar neural correlates when measured by EEG (Daly et al. 2014b). This research hinges on the distinction between perceived and induced emotions, wherein perceived emotions pertain to the understood meaning the listener 
perceives in the music (the compositional intent), and induced emotions are actually conveyed or felt, by the listener (Juslin and Laukka 2004). Thus, a listener may report a piece of music as sounding "sad" but in fact enjoy listening to it.

With recent advances in affective response measurement, for example in determining neurophysiological correlates of affect (Mühl et al. 2015), it appears that the distinction between perceived and induced emotion is a challenge which BCI may help to address in this musical context. In a visual context or multimodal context several systems harness this potential in a variety of BCI for arts systems - see (Gurkok and Nijholt 2013) for a summary of systems including audification, musification, instrument control and emotional expression through BCI art. While visual examples can help differentiate aesthetic responses, music offers perhaps one of the strongest ways to explore this affective phenomenon. The temporal nature of music also lends itself well to the illustration of the changing pattern and transient nature of emotions, and many neurophysiological responses in general. The paradox between enjoyment, perception, and emotional induction has been well explored in musicological research (Hunter et al. 2010; Huron 2011; Manuel 2005) and would be a logical area for further exploration given the startling advances in BCI technology for estimation of affective state; such applications are uniquely afforded by BCI - for example if used to generate music that gradually improves the mood of the patient in an autonomic process without the need for a therapist (Daly et al. 2014b, 2016b).

\section{n.2.3 An example system: MINDMIX, a hybrid BCI interface for music production}

There are many reasons why audio engineers prefer tactile control of mixing processes (Merchel et al. 2010), which partially explains the significant interest, and progress being made in the field of haptic augmentation in audio and musical instrument design (Picinali and Katz 2010; Merchel et al. 2012). 
MINDMIX is a hybrid system (combining active and passive control) using EEG metrics in a many-many mapping of to parameters on an audio mixer by generation of synchronous MIDI Machine Control messages. In this case, end-users might have little or no experience of music mixing, and a such careful mapping to ensure agency and congruence between neurophysiological metric and music parameter is vital. The general methodology for design and application might be equally suited to a wide variety of artistic applications.

In this case the application is ultimately to facilitate control of music production apparatus. Previous attempts to use BCI to control audio mixing parameters have been designed to use alpha and beta activity to control the amplitude of two separate faders (Eduardo R. Miranda 2010). In the case of music mixing, there are many application-specific goals that need to be considered. In, for example, a music therapy context, one advantage of a BCI system is that it might be used by a person with no a priori experience or musical training, in order to engage in music production in context. However, in order to do this the BCI must be capable of performing music which is well correlated with the signal being analysed as a control signal (e.g., BCI parameters mapped according to constraints of melody, harmony, rhythm, or genre) yet also allows the user enough degrees of freedom to feel that they are truly the agent of their performance. The challenge, then, is in devising and evaluating mappings which are most suited to task-specific control - in this case, audio engineering processes, more specifically, mixing processes. MINDMIX control mappings were selected according to this philosophy. For example, once a particular channel has been selected, left or right motor imagery can be actively engaged to adjust the panorama of an audio source to move a sound image between left and right loudspeakers in a 2-channel stereo configuration. This is a many-many mapping wherein the channel is first selected by means of SSVEP, then the pan control selected by ERP, before the pan value is adjusted according to $\mathrm{Mu} \mathrm{L} / \mathrm{R}$ balance. The range of tactile functions the MINDMIX prototype aims to augment are as follows: 
Transport control (play, stop, fwd, rev), fader select and level (individual channels, buss, and FX return), potentiometer select and adjust (pan, parametric EQ), and channel switching (solo, mute, insert, EQ in/out). Each of these parameters has been mapped to a sequence of actively controllable metrics, combining motor imagery (left and right), SSVEP, and ERP. The MINDMIX prototype focusses solely on mixing (including remixing, and post-production tasks), rather than on source capture or recording. Combinations of mappings (i.e., manymany mapping) allows for a channel to be selected using SSVEP, followed by a potentiometer (e.g., pan, or semi-parametric EQ frequency/gain) to be selected according to ERP, before the value of the potentiometer itself is set according to imagined motor imagery (i.e., left, or right). SSVEP allows users to make a selection by focusing their gaze on a visual stimulus oscillating at a given rate. As well as initial parameter selection, SSVEP also allows for second level of control by mapping the duration of the gaze with non-linear features, for example amplitude, allowing for a degree of continuous control i.e., after selecting a specific channel the duration of a user's gaze can be used to adjust the fader for the selected channel accordingly. A similar effect could be achieved using eye-tracking in a hybrid system, using duration of gaze as a secondary mapping for amplitude. The parameters which are most useful for broad user participation in terms of transport across the digital audio workstation are play, stop, select, and various level parameters. It is important to consider the most meaningful signal type for each parameter in the mapping; some of these control signals have analogous actions in a mixer, for example, motor cortex with transport controls (stop, go, fast forward, rewind), and some have analogous parameters in music (SSVEP to non-linear adjustment of amplitude via faders).

Beyond encouraging inclusivity and participation through facilitating access to audio engineering processes via linear mapping strategies, the potential to harness unconscious processes (passive control) suggests that augmented audio engineering, for example, 
individually adaptive, responsive, or context-dependent remixing, may be a possibility. Such technology could be married together with the significant advances in music information retrieval (MIR), non-linear music creation (Berndt 2009), and context-adaptive music selection in the future. For example, creating systems for unsupervised music selection based on individual preferences and brain activity. Of most concern to the prototype described here is the appropriateness of the mapping and the relevance and usefulness of the user interaction with the application. Established methodologies for the evaluation of these types of systems are few. In the traditional audio engineering domain, this would be comparable to evaluating decisions such as whether, for example, a rotary potentiometer or a fader was most appropriate for control of a discrete audio parameter. The remaining sections of this chapter will consider appropriate methods for evaluating musical expression and the design of congruent musical parameter mapping with BCI derived control metrics.

\section{n.3 Musical expression: challenges}

Evaluation of creative computing generally is challenging, and in the case of music, highly context dependent (experience, history, timing, memory, and a whole host of other multimodal factors are involved in experiential evaluation of music). However, a common thread can be drawn between system design across creative computing applications, including music use cases. In order for the performer to feel engaged with the system there must be a sense of agency, which in the case of BCI for music is imbued by the aforementioned parameter mappings. Put plainly, we want the user to feel like a performer, to have some sense of active control over the ensuing musical interaction. The mapping between neurophysiological cues and audio parameter must be intuitive for a neophyte audience (i.e., one without prior training or the physical skills developed by professional audio engineers when working with tactile interfaces). 
In the case of performance, the dream of many musicians, particularly musicians who also engage in composition activity, is to be able to bypass the physical intermediary in the process; that of notation or transcribing ideas for performance. Highly talented musicians are able to do this to some extent when creating and simultaneously performing (the process of musical improvisation). However, this requires a significant degree of musical training and becomes infinitely more complex when other musicians are also involved Those readers who have played musical instruments in isolation will likely find it axiomatic that in the process of collaboration, BCI for music might find a true niche as a viable and meaningful alternative to traditional paradigms. Again, the world of BCI for art has already made significant progress here with examples including work by (De Smedt and Menschaert 2012; Casey and Smith 2013; Lee and Lee 2014), amongst others, which designers and practitioners creating music systems might look to for inspiration.

\section{n.4 Evaluation strategies from auditory display}

A number of paradigms for the evaluation of BCI systems exist, however they often focus on technical or methodological details. There is a tendency in BCI work to prioritise technical implementation in research reporting, for example considering increased speed or accuracy of a system, rather than the application itself. For the purposes of work combining BCI with music, such evaluations are less relevant. In the design of such systems, it is important to consider the most meaningful signal type for each parameter in the mapping; some of the most common BCI control signals have analogous actions in a music performance, for example, motor cortex with physical actions (dancing, tempo, time signature, or starting and stopping an action), and some have analogous auditory parameters (dynamic control of instrument volume for example with amplitude of a frequency band in EEG). However, partly due to the infancy of the use of BCI for music making, the selection of these 
combinations is problematic and tends to become a question of 'taste'. The challenge, then, is in devising evaluation strategies for meaningful mappings which are most suited to taskspecific control, in our case, aesthetic control of music parameters. Established methodologies for the evaluation of these types of systems are few, but borrowing from the world of sonification, multi-criteria decision aid analysis might be a particularly useful paradigm to explore the aesthetic success of a BCI music system, having previously been utilized in data-music mapping strategies.

\section{n.5 Concluding remarks}

This chapter has attempted to give the reader a sense of the possible applications for music performance which the power of BCI might afford. The science fiction scenario is that a listener might 'imagine' a piece of music and through the use of BCI hear that piece realized. Readers of this book will be familiar with the reality will - rightly - be more sceptical, but nevertheless there appears to be a significant opportunity to explore the use of BCI to evaluate systems for creativity - especially complex mechanisms involving multimodal reponses, such as music and the arts - in ways which traditional psychometric profiling might not otherwise offer. The possibilities for audience engagement with music, including emotional communication, physical motility, mood contagion, most importantly, interaction, are well placed as creative examples for BCI which the vast majority of the population might find interesting, even though such systems do not tend to contribute directly to the advancement of BCI technologically, as they are typically problems of engineering implementation rather than advancement. Evaluation strategies for BCI-to-music mappings, in general, are far from universally agreed upon and remain a significant area for further work. One approach would be to borrow from the world of auditory display the use of multi- 
criteria decision aid analysis technique to the evaluation of aesthetic success. In any case, a significant amount of further work remains in quantifying listener responses to music in terms of emotional or experiential communication, such as measurement of impact on induced emotional state versus perceived or self reported emotional state, as traditional psychological approaches suggest that individual preferences and other environmental factors such as cultural expectations and musical training make emotional responses to musical stimuli highly variable (Scherer 2004).

An exciting area of BCI work which this chapter has not explored is the possibility of joint studies combining other neuroimaging techniques, for example fMRI and EEG. For music, such work will be particularly useful, given the spatial resolution with EEG, and the temporal restrictions with fMRI (which make feature correlation from dynamic stimuli such as music listening more challenging, as musical features can change radically over a comparatively short period of time, certainly smaller than the typical frame sizes afforded by fMRI studies). There are practical implications given the size and cost of such facilities but the potential for design of affectively adaptive systems in an artistic context, using such an apparatus, is hugely enticing. The possibility of developing affectively responsive BCI following rigorous evaluation of musical parameter mapping to neural correlates suggests that individual musical interactions might be facilitated by BCI technology in ways that had previously been thought impossible by music technologists, instrument designers, and music psychologists. We have presented a prototype here in $M I N D M I X$ - a mapping between active EEG control and a series of music production (mixing) tasks. Such technology could be married together with the significant advances in music information retrieval (MIR), non-linear music creation, and context-adaptive music selection in the future. For example, creating systems for unsupervised music selection based on individual preferences and brain activity. 


\section{References}

Aldridge, David. 2005. Music therapy and neurological rehabilitation: Performing health. Jessica Kingsley Publishers.

AlZoubi, Omar, Rafael A. Calvo, and Ronald H. Stevens. 2009. Classification of EEG for affect recognition: an adaptive approach. In Australasian Joint Conference on Artificial Intelligence, 52-61. Springer.

AlZoubi, Omar, Irena Koprinska, and Rafael A. Calvo. 2008. Classification of braincomputer interface data. In Proceedings of the 7th Australasian Data Mining ConferenceVolume 87, 123-131. Australian Computer Society, Inc.

Babiloni, Claudio, Fabrizio Vecchio, Francesco Infarinato, Paola Buffo, Nicola Marzano, Danilo Spada, Simone Rossi, Ivo Bruni, Paolo M Rossini, and Daniela Perani. 2011. Simultaneous recording of electroencephalographic data in musicians playing in ensemble. cortex 47: 1082-1090.

Baier, Gerold, Thomas Hermann, and Ulrich Stephani. 2007a. Event-based sonification of EEG rhythms in real time. Clinical Neurophysiology 118: 1377-1386.

Baier, Gerold, Thomas Hermann, and Ulrich Stephani. 2007b. Multi-channel sonification of human EEG. In Proceedings of the 13th International Conference on Auditory Display. Baier, Gerold, Thomas Hermann, and Ulrich Stephani. 2007c. Event-based sonification of EEG rhythms in real time. Clinical Neurophysiology 118: 1377-1386.

Bailes, Freya, and Roger T Dean. 2009. Listeners discern affective variation in computergenerated musical sounds. Perception 38: 1386-1404. doi:10.1068/p6063.

Berndt, Axel. 2009. Musical Nonlinearity in Interactive Narrative Environments. Ann Arbor, MI: MPublishing, University of Michigan Library.

Bigand, Emmanuel, and Bénédicte Poulin-Charronnat. 2006. Are we "experienced listeners"? 
A review of the musical capacities that do not depend on formal musical training. Cognition 100: $100-130$.

Brouwer, Anne-Marie, and Jan van Erp. 2010. A tactile P300 brain-computer interface. Frontiers in Neuroscience. doi:10.3389/fnins.2010.00019.

Casey, Karen, and Damian Smith. 2013. Global mind field-a cybernetic perspective.

Chanel, G., K. Ansari-Asl, and T. Pun. 2007. Valence-arousal evaluation using physiological signals in an emotion recall paradigm. In Systems, Man and Cybernetics, 2007. ISIC. IEEE International Conference on, 2662-2667. doi:10.1109/ICSMC.2007.4413638.

Chanel, G., J. Kronegg, D. Grandjean, and T. Pun. 2006. Emotion assessment: Arousal evaluation using EEG's and peripheral physiological signals. Multimedia Content Representation, Classification and Security: 530-537.

Chew, Yee Chieh (Denise), and Eric Caspary. 2011. MusEEGk: a brain computer musical interface. In Proceedings of the 2011 annual conference extended abstracts on Human factors in computing systems, 1417-1422. New York, N.Y.: ACM Press. doi:10.1145/1979742.1979784.

Clair, Alicia Ann, and Jenny Memmott. 2008. Therapeutic uses of music with older adults. ERIC.

Daly, Ian, James Hallowell, Faustina Hwang, Alexis Kirke, Asad Malik, Etienne Roesch, James Weaver, Duncan Williams, Eduardo Miranda, and Slawomir J Nasuto. 2014. Changes in music tempo entrain movement related brain activity. In 2014 36th Annual International Conference of the IEEE Engineering in Medicine and Biology Society, 4595-4598. IEEE. Daly, Ian, Asad Malik, Faustina Hwang, Etienne Roesch, James Weaver, Alexis Kirke, Duncan Williams, Eduardo Miranda, and Slawomir J Nasuto. 2014. Neural correlates of emotional responses to music: an EEG study. Neuroscience letters 573: 52-57. Daly, Ian, Asad Malik, James Weaver, Faustina Hwang, Slawomir J. Nasuto, Duncan 
Williams, Alexis Kirke, and Eduardo Miranda. 2015. Towards human-computer music interaction: Evaluation of an affectively-driven music generator via galvanic skin response measures. In , 87-92. IEEE. doi:10.1109/CEEC.2015.7332705.

Daly, Ian, Duncan Williams, Faustina Hwang, Alexis Kirke, Asad Malik, Etienne Roesch, James Weaver, Eduardo Miranda, and Slawomir J Nasuto. 2014. Brain-computer music interfacing for continuous control of musical tempo.

Daly, Ian, Duncan Williams, Alexis Kirke, James Weaver, Asad Malik, Faustina Hwang, Eduardo Miranda, and Slawomir J Nasuto. 2016a. Affective brain-computer music interfacing. Journal of Neural Engineering 13: 46022-46035.

Daly, Ian, Duncan Williams, Alexis Kirke, James Weaver, Asad Malik, Faustina Hwang, Eduardo Miranda, and Slawomir J Nasuto. 2016b. Affective brain-computer music interfacing. Journal of Neural Engineering 13: 46022-46035.

De Smedt, Tom, and Lieven Menschaert. 2012. VALENCE: affective visualisation using EEG. Digital Creativity 23: 272-277.

Eaton, Joel, Duncan Williams, and Eduardo Miranda. 2014. Affective Jukebox: A confirmatory study of EEG emotional correlates in response to musical stimuli.

Eaton, Manford L. 1971. Bio-music: biological feedback experimental music systems. Orcus. Fagen, Trudy Shulman. 1982. Music therapy in the treatment of anxiety and fear in terminal pediatric patients. Music Therapy 2: 13-23.

Franco, Fabia, Joel S Swaine, Shweta Israni, Katarzyna A Zaborowska, Fatmata Kaloko, Indu Kesavarajan, and Joseph A Majek. 2014. Affect-matching music improves cognitive performance in adults and young children for both positive and negative emotions. Psychology of Music 42: 869-887.

Goudeseune, Camille. 2002. Interpolated mappings for musical instruments. Organised Sound 7: 85-96. 
Grierson, M. 2008. Composing with brainwaves: minimal trial P300b recognition as an indication of subjective preference for the control of a musical instrument. In Proceedings of International Cryogenic Materials Conference (ICMC'08).

Grierson, Mick, and Chris Kiefer. 2011. Better brain interfacing for the masses. In, 1681. ACM Press. doi:10.1145/1979742.1979828.

Gurkok, Hayrettin, and Anton Nijholt. 2013. Affective brain-computer interfaces for arts. In Affective Computing and Intelligent Interaction (ACII), 2013 Humaine Association Conference on, 827-831. IEEE.

Hanser, Suzanne B. 1985. Music therapy and stress reduction research. Journal of Music Therapy 22: 193-206.

Hinterberger, Thilo, and Gerold Baier. 2005. Poser: Parametric orchestral sonification of eeg in real-time for the self-regulation of brain states. IEEE Trans. Multimedia 12: 70. Hunt, Andy, and Ross Kirk. 2000. Mapping strategies for musical performance. Trends in Gestural Control of Music 21: 231-258.

Hunter, Patrick G, E Glenn Schellenberg, and Ulrich Schimmack. 2010. Feelings and perceptions of happiness and sadness induced by music: Similarities, differences, and mixed emotions. Psychology of Aesthetics, Creativity, and the Arts 4: 47.

Huron, David. 2011. Why is sad music pleasurable? A possible role for prolactin. Musicae Scientiae 15: 146-158.

Juslin, Patrik N., and Petri Laukka. 2004. Expression, perception, and induction of musical emotions: A review and a questionnaire study of everyday listening. Journal of New Music Research 33: 217-238.

Kirke, Alexis, Eduardo Miranda, and Slawomir J. Nasuto. 2013. Artificial Affective Listening towards a Machine Learning Tool for Sound-Based Emotion Therapy and Control. In Proceedings of the Sound and Music Computing Conference, 259-265. Stockholm, 
Sweden: SMC Network.

Kirke, Alexis, Eduardo Reck Miranda, and Slawomir Nasuto. 2012. Learning to Make Feelings: Expressive Performance as a part of a machine learning tool for sound-based emotion therapy and control. In Cross-Disciplinary Perspectives on Expressive Performance Workshop. London.

Knapp, R. Benjamin, Javier Jaimovich, and Niall Coghlan. 2009. Measurement of motion and emotion during musical performance.

Knapp, R. Benjamin, and Hugh S. Lusted. 1990. A bioelectric controller for computer music applications. Computer music journal 14: 42-47.

Le Groux, S., and P. Verschure. 2009. Neuromuse: Training your brain through musical interaction. In Proceedings of the International Conference on Auditory Display, Copenhagen, Denmark.

Lee, Hae Young, and Won Hyung Lee. 2014. A study on interactive media art to apply emotion recognition. International Journal of Multimedia \& Ubiquitous Engineering 9: 12. Leslie, G., and T. Mullen. 2012. MoodMixer: EEG-based Collaborative Sonification. In Proceedings of the International Conference on New Interfaces for Musical Expression\$ \$, 296-299. http://www.nime.org/proceedings/2011/nime2011_296.pdf. Accessed November 19.

Lin, Chih-Yi, and Stone Cheng. 2012. Multi-theme analysis of music emotion similarity for jukebox application. In Audio, Language and Image Processing (ICALIP), 2012 International Conference on, 241-246. IEEE.

Lin, Yuan-Pin, Chi-Hong Wang, Tzyy-Ping Jung, Tien-Lin Wu, Shyh-Kang Jeng, Jeng-Ren Duann, and Jyh-Horng Chen. 2010. EEG-Based Emotion Recognition in Music Listening. IEEE Transactions on Biomedical Engineering 57: 1798-1806. doi:10.1109/TBME.2010.2048568. 
Lucier, Alvin. 1976. Statement on: music for solo performer. Biofeedback and the Arts, Results of Early Experiments. Vancouver: Aesthetic Research Center of Canada Publications: 60-61.

Lyon, Eric, R. Benjamin Knapp, and Gascia Ouzounian. 2014. Compositional and performance mapping in computer chamber music: A case study. Computer Music Journal. Manuel, Peter. 2005. Does sad music make one sad? An ethnographic perspective. Contemporary Aesthetics 3.

Manzolli, J., and P. F. M. J. Verschure. 2005. Roboser: A real-world composition system. Computer Music Journal 29: 55-74.

Merchel, Sebastian, Ercan Altinsoy, and Maik Stamm. 2010. Tactile Music Instrument Recognition for Audio Mixers. In Audio Engineering Society Convention 128. Merchel, Sebastian, M. Ercan Altinsoy, and Maik Stamm. 2012. Touch the sound: audiodriven tactile feedback for audio mixing applications. Journal of the Audio Engineering Society 60: 47-53.

Middendorf, Matthew, Grant McMillan, Gloria Calhoun, Keith S. Jones, and others. 2000. Brain-computer interfaces based on the steady-state visual-evoked response. IEEE Transactions on Rehabilitation Engineering 8: 211-214.

Miranda, Eduardo R. 2010. Plymouth brain-computer music interfacing project: from EEG audio mixers to composition informed by cognitive neuroscience. International Journal of Arts and Technology 3: 154-176.

Miranda, Eduardo Reck, and Julien Castet, ed. 2014. Guide to brain-computer music interfacing. London: Springer.

Mühl, Christian, Dirk Heylen, and Anton Nijholt. 2015. Affective brain-computer interfaces: neuroscientific approaches to affect detection. In Oxford Handbook of Affective Computing, 217-232. Oxford University Press Oxford. 
Mullen, Tim, Alexander Khalil, Tomas Ward, John Iversen, Grace Leslie, Richard Warp, Matt Whitman, et al. 2015. MindMusic: playful and social installations at the interface between music and the brain. In More Playful User Interfaces, 197-229. Springer. Nirjon, Shahriar, Robert F Dickerson, Qiang Li, Philip Asare, John A Stankovic, Dezhi Hong, Ben Zhang, Xiaofan Jiang, Guobin Shen, and Feng Zhao. 2012. Musicalheart: A hearty way of listening to music. In Proceedings of the 10th ACM Conference on Embedded Network Sensor Systems, 43-56. ACM.

Pérez, Miguel Angel Ortiz, and R. Benjamin Knapp. 2008. BioTools: a biosignal toolbox for composers and performers. In Computer Music Modeling and Retrieval. Sense of Sounds, 441-452. Springer.

Picinali, Lorenzo, and B. F. Katz. 2010. Spectral discrimination thresholds comparing audio and haptics. In Proceedings of haptic and auditory interaction design workshop, Copenhagen, 1-2.

Ramirez, Rafael, and Zacharias Vamvakousis. 2012. Detecting Emotion from EEG Signals Using the Emotive Epoc Device. In Brain Informatics, ed. FabioMassimo Zanzotto, Shusaku Tsumoto, Niels Taatgen, and Yiyu Yao, 7670:175-184. Lecture Notes in Computer Science. Springer Berlin Heidelberg.

Rosenboom, David. 1990. The performing brain. Computer Music Journal 14: 48-66. Russell, J. A. 1980. A circumplex model of affect. Journal of personality and social psychology 39: 1161.

Scherer, K. R. 2004. Which emotions can be induced by music? What are the underlying mechanisms? And how can we measure them? Journal of new music research 33: 239-251. Snyder, Joel S., and Edward W. Large. 2005. Gamma-band activity reflects the metric structure of rhythmic tone sequences. Cognitive Brain Research 24: 117-126. doi:10.1016/j.cogbrainres.2004.12.014. 
Teitelbaum, Richard. 1976. In tune: Some early experiments in biofeedback music (19661974). Biofeedback and the Arts, Results of Early Experiments. Vancouver: Aesthetic Research Center of Canada Publications.

Toharia, Pablo, Juan Morales, Octavio Juan, Isabel Fernaud, Angel Rodríguez, and Javier DeFelipe. 2014. Musical Representation of Dendritic Spine Distribution: A New Exploratory Tool. Neuroinformatics: 1-13. doi:10.1007/s12021-013-9195-0.

Väljamäe, A., T. Steffert, S. Holland, X. Marimon, R. Benitez, S. Mealla, A. Oliveira, and S. Jordà. 2013. A review of real-time EEG sonification research.

Vuoskoski, Jonna K., and Tuomas Eerola. 2012. Can sad music really make you sad? Indirect measures of affective states induced by music and autobiographical memories. Psychology of Aesthetics, Creativity, and the Arts 6: 204.

Vuoskoski, Jonna K., William F. Thompson, Doris McIlwain, and Tuomas Eerola. 2012. Who enjoys listening to sad music and why? Music Perception 29: 311-317. Williams, Duncan. 2016. Utility Versus Creativity in Biomedical Musification. Journal of Creative Music Systems 1.

Wu, Dan, Chaoyi Li, Yu Yin, Changzheng Zhou, and Dezhong Yao. 2010. Music composition from the brain signal: representing the mental state by music. Computational intelligence and neuroscience 2010: 14. 